УдК 621.391:621.396

\title{
ОЦЕНИВАНИЕ ПАРАМЕТРОВ ПАССИВНЫХ ПОМЕХ НА ОСНОВЕ КОСВЕННЫХ АЛГОРИТМОВ
}

\author{
Д. И. ПОПОВ \\ Рязанский государственный радиотехнический университет, \\ Россия, Рязань, 390005, ул. Гагарина, д. 59/1
}

\begin{abstract}
Аннотация. Методом максимального правдоподобия синтезированы косвенные алгоритмы оценивания параметров пассивных помех, использующие линейные преобразования исходных данных. Введена функция правдоподобия для преобразованных в соответствии с суммарно-разностными алгоритмами входных отсчетов. Полученные в результате решения соответствующих уравнений правдоподобия алгоритмы оценивания коэффициентов межпериодной корреляции и доплеровского сдвига фазы не содержат традиционной операции комплексного перемножения входных данных. Приведена структурная схема измерителя оценок соответствующих параметров пассивной помехи, которые могут быть использованы в адаптивных режекторных фильтрах. Проведен анализ точности оценивания искомых параметров пассивной помехи в зависимости от объема обучающей выборки и корреляционных свойств помехи. Сравнение результатов моделирования и расчетов для косвенных и прямых алгоритмов показало их полное совпадение и подтвердило асимптотическую эффективность получаемых оценок и эквивалентность косвенных и прямых алгоритмов.
\end{abstract}

Ключевые слова: алгоритм оценивания; метод максимального правдоподобия; суммарно-разностный алгоритм; коэффициент корреляции; доплеровский сдвиг фазы; пассивная помеха; точность оценивания

\section{ВВЕДЕНИЕ}

Априорная неопределенность спектрально-корреляционных характеристик пассивных помех, создаваемых мешающими отражениями от неподвижных или медленно перемещающихся объектов, а также их неоднородность и нестационарность в зоне обзора существенно затрудняют реализацию эффективного обнаружения движущихся целей на фоне пассивных помех $[1,2]$. Преодоление априорной неопределенности параметров помехи в соответствии с методологией адаптивного байесовского подхода основывается на замене неизвестных параметров помехи их состоятельными оценками, что приводит к построению адаптивных алгоритмов и систем обработки, основным узлом которых являются адаптивные режекторные фильтры [3, 4].

Одной из основных задач, возникающих при адаптивном режектировании пассивных помех, является оценивание неизвестных параметров помехи - аргумента и модуля коэффициентов корреляции. Оптимальные алгоритмы и устройства оценивания этих параметров могут быть синтезированы прямым использованием метода максимального правдоподобия [5- 12]. Однако в ряде случаев на практике реализация присущей оптимальным алгоритмам операции комплексного перемножения входных данных при условии работы измерителей в реальном масштабе времени оказывается затруднительной, что приводит к косвенным ал- 


\section{БИБЛИОГРАФИЧЕСКИЙ СПИСОК}

1. Skolnik, M. I. (ed.). Radar Handbook, 3rd ed. McGraw-Hill, 2008. 1352 p.

2. Richards, M. A.; Scheer, J. A.; Holm, W. A. (eds.). Principles of Modern Radar: Basic Principles. New York: SciTech Publishing, IET, Edison, 2010. 924 p.

3. Попов, Д.И. “Адаптация нерекурсивных режекторных фильтров,” Известия вузов. Радиоэлектроника, Т. 52, № 4, C. 46-55, 2009. URI: http://radio. kpi.ua/article/view/S0021347009040050.

4. Лозовский, И.Ф. "Построение и эффективность адаптивной обработки сигналов в условиях воздействия комбинированных помех," Успехи современной радиоэлектроники, № 1, C. 52-58, 2016. URI: http://www.radiotec.ru/article/7230.

5. Melvin, W. L.; Scheer, J. A. (eds.). Principles of Modern Radar: Advanced Techniques. New York: SciTech Publishing, IET, Edison, 2013. 846 p.

6. Richards, M. A. Fundamentals of Radar Signal Processing, 2nd ed. New York: McGraw-Hill Education, 2014. $618 \mathrm{p}$.

7. Popov, D. I.; Smolskiy, S. M. "Estimation of the clutter correlation coefficient in radar systems," Infocommun. J., Vol. VIII, No. 3, p. 8-12, Sept. 2016. 
8. Perozzi, G.; Efimov, D.; Biannic, J.-M.; Planckaert, L.; Coton, P. "Wind estimation algorithm for quadrotors using detailed aerodynamic coefficients," Proc. of Annual American Control Conf., ACC, 27-29 Jun. 2018, Milwaukee, USA. IEEE, 2018, p. 1921-1926. DOI: 10.23919/ACC.2018.8431879.

9. Malikov, E.; Sun, Y. "Semiparametric estimation and testing of smooth coefficient spatial autoregressive models," J. Econometrics, Vol. 199, No. 1, p. 12-34, 2017. DOI: 10.1016/j.jeconom.2017.02.005.

10. Tarima, S. "Statistical estimation in the presence of possibly incorrect model assumptions," J. Statistical Theory Practice, Vol. 11, No. 3, p. 449-467, 2017. DOI: 10.1080/15598608.2017.1299056.

11. Karmakar, B.; Mukhopadhyay, I. "Risk efficient estimation of fully dependent random coefficient autoregressive models of general order," Commun. Statistics - Theory Methods, Vol. 47, No. 17, p. 4242-4253, 2018. DOI: $\underline{10.1080 / 03610926 .}$ 2017.1371758.

12. Sun, Y.; Malikov, E. "Estimation and inference in functional-coefficient spatial autoregressive panel data models with fixed effects," J. Econometrics, Vol. 203, No. 2, p. 359-378, 2018. DOI: 10.1016/j.jeconom.2017.12.006.

13. Кошевой, В. М.; Медведик, А. Д. “Построение измерителей параметров пассивных помех на основе схем череспериодной компенсации," Известия вузов. Радиоэлектроника, Т. 23, № 12, С. 82-84, 1980.

14. Olguin, C. J. M.; Sampaio, S. C.; dos Reis, R. R. "Statistical equivalence of prediction models of the soil sorption coefficient obtained using different $\log \mathrm{P}$ algorithms," Chemosphere, Vol. 184, p. 498-504, 2017. DOI: 10.1016/j.chemosphere.2017.06.027.

15. Pepelyshev, A.; Kornikov, V.; Zhigljavsky, A. "Statistical estimation in global random search algorithms in case of large dimensions," in: Battiti, R.; Kvasov, D.; Sergeyev, Y. (eds.) Learning and Intelligent Optimization. LION 2017. Lecture Notes in Computer Science, Vol. 10556. Springer, Cham. DOI: 10.1007/978-3-319-69404-7 32.

16. Hua, X.; Cheng, Y.; Wang, H.; Qin, Y. "Information geometry for covariance estimation in heterogeneous clutter with total Bregman divergence," Entropy, Vol. 20, No. 4, p. 258, 2018. DOI: $\underline{10.3390 / \mathrm{e} 20040258 .}$.

17. Lazrieva, N.; Toronjadze, T. "Recursive estimation procedures for one-dimensional parameter of statistical models associated with semimartingales," Trans. A. Razmadze Math. Institute, Vol. 171, No. 1, p. 57-75, 2017. DOI: 10.1016/j.trmi.2016.12.001.

18. Попов, Д. И. “Адаптивное режектирование пассивных помех," Известия вузов. Радиоэлектрони$\kappa a, \quad$ Т. 56, № 4, С. 39-47, 2013. DOI: 10.20535/S0021347013040031. 